\title{
High resolution spectral characteristics of the Earth-ionosphere cavity resonances
}

D. BEAMISH and A. TZANIS

British Geological Survey, Murchison House, West Mains Road, Edinburgh EH9 3LA, U.K.

Beamish, D. \& Tzanis, A., 1986. High resolution spectral characteristics of the Earthionosphere cavity resonances. Journal of Atmospheric and Terrestrial Physics, 48, 187203.

DOI: 10.1029/RS011i010p00803

\section{Abstract}

The natural resonances of the Earth-ionosphere cavity at frequencies between 5 and $100 \mathrm{~Hz}$ have been studied since the fundamental paper by Schumann. While the gross features of the phenomena are now well understood, considerable work remains to be done on their detailed behaviour. In the present study a high resolution, data adaptive spectral technique is applied to digital electromagnetic data obtained at moderate latitude. A particular feature of the method employed is that spectral properties become available on the same time scale as many ELF events, thus both time local and time averaged resonance features can be readily established. The technique can thus be applied to both dynamic and steady-state descriptions of the cavity's properties.

For the data set considered, the technique adequately resolves the first six resonance modes on a time scale of $0.75 \mathrm{~s}$. The presence of higher order modes is also indicated. The time averaged frequencies obtained are in accord with those of previous experimental determinations. When the time local properties of individual transient waveforms are examined, however, we observe a number of detailed effects which are predicted by theory. The precise spectral structure of the resonance modes appears influenced by the differing locations of the sources of transient excitation. In the case of the first order resonance mode, the properties of the cavity consistently support both singlet and doublet resonance behaviour. 


\section{INTRODUCTION}

ELF waves in the Earth-ionosphere waveguide, generated by the global sum of thunderstorm activity, are able to propagate over large distances with very little attenuation (GALEJS, 1964). The waveguide becomes a cavity resonator at such frequencies and for a perfectly reflecting ionosphere the first five resonance modes would be established at frequencies of 10.6, 18.4, 26.0, 35.5 and $41.1 \mathrm{~Hz}$ (MADDEN and THOMSON, 1965).

In reality, due to energy losses, the first five modes have typical lower frequencies observed at 7.8, 14.1,20.3, 26.3 and 32.5 Hz (MADDEN and THOMSON, 1965). These are termed the Schumann Resonances. As pointed out by GALEJS (1961) and by POLK (1969), the relative amplitudes of the resonance peaks in the Schumann power spectrum depend primarily on the source-receiver separation $(\theta)$, while the precise values of the resonance frequencies depend not only on $\theta$, but also on the electrical conductivity of the ionosphere. Thus it is anticipated that the analysis of the Schumann spectrum will permit a description of the world-wide average conductivity profile of the lower ionosphere (TRAN and POLK, 1979a).

The resonant eigenfrequencies established from theory are a function of the complexity of the model considered. In models which treat the ionosphere as spherically symmetric, the eigenvalues of the wave equation are degenerate, giving rise to single, precise eigenfrequencies for the resonant modes. Such degeneracy may be partially or completely removed when azimuthal asymmetries in the waveguide (e.g. the day-night ionospheric inhomogeneity) are taken into account. Under such circumstances, frequency splitting of the fundamental resonant modes may occur (MADDEN and THOMSON, 1965). An additional complication is introduced when the Earth's magnetic field is taken into account. The presence of the magnetic field necessitates the inclusion of gyrotropic waveguide properties and results in different phase velocities for waves travelling in opposite directions around the Earth (BLIOKH et al., 1980). This again has the resultant effect of producing frequency splitting of the resonant modes. In the mathematical development of the field expressions, the Legendre polynomials of degree $\mathrm{n}$ are replaced by the associated Legendre polynomials of degree $(2 n+1)$, causing a $(2 n+1)$ splitting of the resonant frequency. 
BLIOKH et al. (1980) demonstrate such effects of the first $(n=1)$ resonant mode by considering three models. Model (a) consists of a perfect Earth resonator, model (b) consists of an Earth resonator with polar inhomogeneity and model (c) consists of an Earth resonator with a dipole magnetic field. For model (a) the first mode has the degenerate frequency of $f=10.6 \mathrm{~Hz}$. In model $(b)$ the waveguide can support oscillations at two frequencies $f(1)=8 \mathrm{~Hz}(m=0)$ and $f(2)=7.8 \mathrm{~Hz}(m=1)$, where $m$ is the azimuthal wave velocity of the wave assumed to possess an $\exp (\mathrm{imQ})$ azimuthal dependence. In model (c) the $(2 n+1)$ triplet split is observed with the frequencies established at $f(1)=7.5 \mathrm{~Hz}(m=-1$, west to east equatorial propagation), $f(2)=8 \mathrm{~Hz}$ $(m=0)$ and $f(3)=8.16 \mathrm{~Hz}(m=+1)$. The model parameters were chosen to produce a normalized frequency of $8 \mathrm{~Hz}$ for the $\mathrm{m}=0$ mode.

The above models constitute 'steady-state' descriptions of the waveguide properties in which the ionospheric conductivity profile is essentially fixed. Since the ionosphere has dynamic properties, a number of papers detail how the electrical conductivity profile of the lower ionosphere (C-, D- and E-regions) affects the characteristics of the Schumann resonance spectra (MADDEN and THOMSON, 1965;TRAN and POLK, 1979b; SENTMAN, 1983). TRAN and POLK (1976) have shown that the region which determines the resonance frequencies is between 40 and $100 \mathrm{~km}$. Different ionisation mechanisms are responsible for maintaining the electrical conductivity at different altitudes through this interval. lonisation sources include galactic cosmic rays (lower Dor C-region), solar Lyman- $\alpha$ (D-region) and solar X-rays (E-region). Changes in the intensity or availability of such sources therefore modulate the ionospheric conductivity at the corresponding altitude. These variations in turn influence the characteristics of the Schumann resonances. Typically, an increase of conductivity below $63 \mathrm{~km}$ (lower D-region acting as a dielectric) would lower the resonance frequencies, while an increase of conductivity within the upper D-region (acting as an imperfect conductor) would lead to an increase in the resonance frequencies. In the model ionosphere studied by TRAN and POLK (1979a), changes in the conductivity of the $D$ - and lower E-regions led to frequency shifts of $10 \%$ or more in the first three resonance frequencies. TRAN and POLK (1979b) provide a method for evaluating conductivity profiles based on three sets of observational parameters : (a) the exact values of the resonance frequencies; (b) the ratios of the spectral amplitudes at successive resonance peaks ; (c) the bandwidths of the resonance peaks at the $90 \%$ amplitude level.

SENTMAN (1983) has studied the effects on the Schumann resonance frequencies of perturbations in a 'steady-state' exponential ionospheric profile as an aid in 
interpreting observations in terms of the sources of ionisation. The models used provide overall frequency variations of $\pm 1.0, \pm 1.5$ and $\pm 2.5 \mathrm{~Hz}$ for the $n=1,2$ and 3 Schumann resonance modes. In addition, fluctuations in the resonance frequencies from observational data have been studied in relation to the occurrence of sudden ionospheric disturbances, having onset times of the order of minutes (CANNON and RYCROFT, 1982). In all cases it is necessary to establish precise values of the resonance frequencies from experimental data sets over appropriate time windows.

The ELF waves propagate as a $0^{\prime}$ th order TM mode in which the electric field is largely radial. As a consequence, vertical antennas are often used as appropriate omnidirectional receivers of the ELF noise in the audio and subaudio bands. Equally, sensitive magnetometers, such as induction coils, measuring the horizontal components of the magnetic field may also be used to provide equivalent data. Such data sets have been used to estimate resonance frequencies with a temporal resolution (integration time) of $13.6 \mathrm{~min}$ (CANNON and RYCROFT, 1982) and 17and 34 min (TRAN and POLK, 1979b). Such a long time base appears to be required due to the Fourier techniques used to analyse the data sets. Using such data and integration times, the typical frequency resolution is quoted as being $0.156 \mathrm{~Hz}$ (CANNON and RYCROFT, 1982) and $0.125 \mathrm{~Hz}$ (TRAN and POLK, 1979b).

Clearly, any improvement in time and/or frequency resolution would assist in the comparison of models and observations. The purpose of the present paper is to point out the growing availability of digital audio frequency data sets and to demonstrate a high time/frequency resolution spectral technique that can be applied in the analysis of such data. The technique permits adequate frequency resolution over time windows of several seconds for the $\mathrm{n}=1$ mode and less than one second for the higher modes. Since such time windows are of the same order as the duration of many transient ELF 'events' (JONES and KEMP, 1971; BLIOK et al., 1980), the dynamic components of the resonance phenomena are highly resolved and the average, or integrated, properties can be established across relatively short time periods.

\section{AUDIOMAGNETOTELLURIC DATA}

Audiomagnetotelluric (AMT) geophysical investigations provide a means of studying the Earth's electrical conductivity structure at shallow crustal depths. Methodologies are described by KELLER (1971), STRANGWAY et al. (1973) and FISCHER (1982). The basic data set collected at a given location consists of two (usually orthogonal) horizontal telluric channels, measuring time changes in the induced electric field, and 
two components of the horizontal magnetic field. The bandwidth available is governed by the magnetic sensors employed in the field instrumentation. AMT instrumentation nominally covers the frequency range $1-100 \mathrm{~Hz}$. However, the high frequency limit may extend from 10 to $100 \mathrm{KHz}$.

Table 1. Frequency characteristics of the three decade data scheme used in the present study

\begin{tabular}{lcrr}
\hline & $f_{L}$ & $f_{H}$ & $\Delta f$ \\
\hline Decade 1 & 10 & 100 & 400 \\
Decade 2 & 1 & 10 & 40 \\
Decade 3 & 0.1 & 1 & 4 \\
\hline
\end{tabular}

$f_{L}$, low frequency $-3 \mathrm{~dB}$ point $; f_{H}$, high frequency $-3 \mathrm{~dB}$ point; $\Delta f$, data sampling frequency. All frequencies in $\mathrm{Hz}$.

The data described in the present study were acquired with a minicomputer based AMT field system covering the bandwidth $0.01-100 \mathrm{~Hz}$. Band-pass and notch filters are applied to condition the analogue signals prior to 12 -bit digitisation. The conditioning scheme provides the 3 decades and sampling rates shown in Table 1. In relation to the Schumann resonances, decade 2 provides data for studies of the $n=1(8 \mathrm{~Hz})$ mode, while decade 1 provides data for studies of the higher order modes. The two decades are collected across a window of 300 data points. The time interval for the collection of each data window is thus $0.75 \mathrm{~s}$ for decade 1 and $7.5 \mathrm{sfor}$ decade 2 . Data collection between successive data windows is at present discontinuous due to in-field processing and data storage on a slow digital cartridge. Such discontinuities are not a limitation to the subsequent spectral analysis, since the 300 data points sampled at twice the Nyquist frequency provide sufficient degrees of freedom across the bandwidth of each decade. Such temporal resolution is advantageous only if spectral characteristics can be adequately resolved from the number of data points made available in each data window. This question is considered in the following section.

When examining the data on these time scales it is possible that either the resonance waveforms are totally absent, for certain data windows, or that the waveforms exhibit large amplitude variance over successive data windows. Such amplitude variance is common. An example is provided in Fig. 1, which displays decade 1 data $(10-100 \mathrm{~Hz})$ for five successive data windows in two orthogonal telluric (induced electric) and two orthogonal magnetic channels. 
For a single radial source filament, the horizontal magnetic field $(\mathrm{H}<1>$.) generated is proportional to the angular source-receiver separation (GALEJS, 1972) as:

$$
H_{\phi n} \propto \frac{\partial}{\partial \theta} P_{n}(\cos \theta)
$$

where the subscript $\mathrm{n}$ is the resonance mode number and $\mathrm{P} .(\cos 8)$ is the Legendre function of order $\mathrm{n}$. In spectral analysis of any given data window only power magnitudes are required, since the cavity resonance may be excited simultaneously by a multiplicity of incoherent sources. For each thunderstorm centre considered, the lightning spectrum may be represented by the relation (GALEJS, 1961):

$$
g(\omega)=c \times \exp \left(-9.1 \times 10^{-3} \omega\right)
$$

with c a constant and w angular frequency. The received power spectrum, G.(w), will be (POLK, 1969):

$$
G_{n}(\omega)=\int_{\phi_{1}}^{\phi_{2}} \int_{\theta_{1}}^{\theta_{2}} g(\omega)\left|H_{\phi n}\right|^{2} \sin \theta \mathrm{d} \theta \mathrm{d} \phi
$$

for a uniform distribution of $\mathrm{g}(\mathrm{w})$ between the limits 81,82 and $</ \mathrm{J} 1,</ \mathrm{J} 2$. For multiple and distributed thunderstorm centres, the power spectrum received is thus the sum of individual contributions of the form given above. The length of the data windows used in the present study are of the same order as the time scales of the models of lightning discharge (e.g. JONES and KEMP, 1971) and we therefore anticipate large variations in signal/noise ratios.

The data examined in the present study were collected in western Anatolia (Turkey), geographic coordinates $40.5^{\circ} \mathrm{N}, 30^{\circ} \mathrm{E}$. The data were selected purely on the grounds of good signal/noise ratios. The decade 1 and decade 2 data were collected on days 209 and 159,1984 , respectively.

\section{DATA ANALYSIS}

Figure 2 shows typical data recorded in orthogonal electric $(E)$ and magnetic $(H)$ channels and the associated raw (unsmoothed) power spectra for decade 1 data. 
The relatively flat power spectra observed indicates a white noise process. In calculating the power spectrum, and associated confidence limits, using the discrete Fourier transform, it is necessary to smooth adjacent power estimates to reduce variance. If it is assumed that the data consist of a white noise series ( $\mathrm{n}(\mathrm{i})$ ) of length $\mathrm{N}$ .$\Delta t$, the fractional error $\varepsilon$ (the ratio of RMS deviation to mean) in the smoothed spectral estimates is given by:

$$
\varepsilon=\frac{1}{\sqrt{2 m+1}}
$$

when the raw estimates are averaged over $2 \mathrm{~m}+1$ data points (JENKINS and WATTS, 1968). For a given fractional error, the raw spectral estimates must be smoothed over the frequency interval $\Delta \mathrm{f}$ given by:

$$
\Delta f=\frac{1}{2 \cdot N \cdot \Delta t \cdot \varepsilon^{2}}
$$

Thus for a stable estimate of the power spectrum, $\varepsilon$ must clearly be small and the estimate must be smoothed over a wide frequency range. The reliability of the estimate is improved at the expense of frequency resolution unless $\mathrm{N}$ is made large.

If the time series is of autoregressive form, i.e.

$$
n_{i}+\sum_{m=1}^{M} a_{m} n_{i-m}=x_{i}
$$

where $x_{i}$ is a white noise series, it is possible to improve the resolution beyond the above limits for a given $N$. The property of the filter $(a(m), m=1, M)$ that generates the autoregressive time series is determined by a small number of filter coefficients. The filter coefficients can be determined using a finite portion of the autocorrelation function $R_{m}=\langle n(i) n(i+m)>$ for $m=0, M-1$ and where the brackets denote ensemble averages. The power spectrum can then be determined from the filter coefficients obtained. This is the basis of the maximum entropy method of spectral analysis (e.g. ULRYCH and BISHOP, 1975).

A number of methods exist for estimating the autoregressive (AR) filter coefficients. Among these the algorithm due to BURG (1968) is both data adaptive and 
computationally efficient. The highest order filter coefficient (reflection coefficient) is determined by minimizing the error power output from the filter fitted to the data in both time directions. The remaining coefficients are calculated using the LEVINSON (1947) recursive algorithm, which involves previously determined lower order filters. The procedure is iterative and fits successively higher order autoregressive operators to the data until a specified order is reached or a cut-off criterion is satisfied. This procedure ensures that the filter is minimum delay, and therefore stable. When referring to ME spectra we henceforth implicitly refer to Burg's algorithm.

Once the AR coefficients are determined, the power spectrum can be obtained through the inverse of the $z$-transform of the filter scaled by the minimum residual power of the input time series. The advantage of the power spectrum so derived is the inherent frequency resolution, which is superior to any of the standard spectral analysis techniques. It is worth noting that in the case of ME spectra, it is the area under a given spectral peak that determines the power level, rather than the actual peak height.

A major problem that arises in the use of the ME method is the determination of the order of the AR process, i.e. the number of filter coefficients $(M)$ that are sufficient to describe the process. If $\mathrm{M}$ is too small, the data are underfitted, a smooth spectrum will result and the high resolution capability of the method is lost. If $\mathrm{M}$ is too large and the data are overfitted, undesired effects may occur. This problem has led to a number of criteria to establish the correct order of the AR process. The most widely used theoretical criteria are Akaike's final prediction error (FPE) criterion, (AKAIKE, 1969, 1970), Akaike's theoretical information criterion (AIC) (AKAIKE, 1974, 1976) and Parzen's criterion for autoregressive transfer functions (CAT) (PARZEN, 1976). Other empirical criteria have been proposed where the above fail or seem to be inconclusive. The empirical criteria suggest limiting the order of the filter length (M) to a given fraction of the data length (N). ULRYCH and CLAYTON (1976) propose a filter length of between $N / 2$ and N/3, while BERRYMAN (1978) proposes a length of $2 \mathrm{~N} / \ln (2 \mathrm{~N})$.

For the present study, use was made of Akaike's FPE and AIC criteria. The behaviour of the AR filter as monitored by the performance of these criteria shows a high degree of variability between data windows. Figure $3 a$ shows the results from six typical data windows displaying the variation of FPE and AIC, normalized to unity, against the order of the $A R$ process, i.e. the number of filter coefficients $M$, where $M$ ranges from 1 to 100. The data length in each case is $N=150$. It can be seen that the normalized FPE 
and AIC parameters display a series of local minima, rather than the expected monotonically decreasing character, while the absolute minimum displays large variability in terms of the order of the AR model between the six data windows.

Because of the poor performance of the theoretical criteria, the selection of the order of the AR model was necessarily carried out semi-empirically. Clearly, the order of filter that will adequately resolve at least all the spectral peaks predicted by theory and that will not overfit the data with the associated undesired effects on the majority of the data windows can be defined to be optimum for our present purposes. Given the large number of data runs available, it is possible to examine the probability distribution of the position of the absolute minimum of the FPE as a function of $M$, the order of the AR model. Figure $3 \mathrm{~b}$ displays the frequency of occurrence of the absolute minimum as a function of $\mathrm{M}$, using 150 data windows of decade 1 and decade 2 data. The distributions obtained display different characteristics for the two decades. For decade 2 data the position of the absolute minimum occurs for the majority of data windows in the interval $\mathrm{M}=10-25$. For decade 1 data the equivalent interval is $\mathrm{M}=25-35$.

Filter lengths of $M=12,18,25$ and 35 have been implemented with all data lengths set at $\mathrm{N}=150$. The maximum filter length used is in fact less than any of the empirical criteria quoted above. The spectral resolution available using such filter lengths is illustrated in Fig. 4. The average spectrum normalized to unity, has been obtained from 25 successive data windows using $M=12,18$ and 25 for decade 2 (Fig. 4a) and decade 1 (Fig.4b). We retain a spectral resolution of $1.0 \mathrm{~Hz}$ (decade 1) and $0.1 \mathrm{~Hz}$ (decade 2), throughout. It can be seen in Fig. 4a that the 7-8 Hz peak obtained for $\mathrm{M}=12$ decompose to two stable peaks for higher order lengths. In Fig. $4 \mathrm{~b}$ it is observed that a full complement of the higher order Schumann resonance modes is only established using a filter of length $M=25$. In view of these results and those presented in Fig. $3 \mathrm{~b}$, filter lengths of $\mathrm{M}>40$ (i.e. $>26 \%$ of the data length) do not appear warranted for the present data. Specifically, for the examination of time averaged properties we consider optimum filter lengths to be $M=18$ for decade 2 data and $M=25$ for decade 1 data. For the examination of time local properties (e.g. sonograms) higher order filter lengths of $\mathrm{M}=25$ (decade 2 ) and $\mathrm{M}=35$ (decade 1 ) are required and have been implemented.

The term 'undesired effects' in the ME literature is understood to mean non-physical line splitting and frequency shifting of spectral peaks. Such phenomena have been the 
subject of rigorous investigation by several authors, as they limit the performance of the ME method for certain types of data. JAYNES (1982) has shown that such effects occur during the analysis of 'circular' time series, in which the independence of consecutive data windows is reduced by the circularity of the process. This is simulated as phase jumps that occur at regular time intervals, and is manifested by the appearance of multiple spectral lines close to the nominal frequency. Furthermore, as FOUGERE (1977) points out, such effects occur in the low noise case and are unique to the ME method. It is therefore anticipated that such problems arise during the analysis of slowly varying phenomena of low order processes with moderate to large signalto-noise ratios, such as, for instance, artificial time series of sinusoids in noise, with variable initial phases. Our data, in general, possess none of the above qualities. However, rigorous comparisons have been carried out of the peak frequencies resolved by the ME and FFT methods, with the assistance of theory for control, and we are satisfied that the ME method used in the present analysis produces no substantial undesired effects for the filter lengths implemented.

\section{RESULTS}

Each ME spectrum is obtained from individual data windows of 150 points. The time intervals are $0.75 \mathrm{~s}$ for decade $1(10-100 \mathrm{~Hz})$ and $7.5 \mathrm{~s}$ for decade $2(1-10 \mathrm{~Hz})$ data. Successive data windows are separated by approximately $3 \mathrm{~s}$. As shown in Fig. 5, the H $(\mathrm{N}-\mathrm{S})$ magnetic component displays the largest resonance power level of the 4 component data and this component is therefore used to display the Schumann resonance characteristics.

The results are first considered as a series of evolutionary spectra from successive data windows. Each spectrum is plotted on a linear frequency scale and has been normalized to a peak value of unity to allow for the variance in waveform amplitudes. In addition, as successive data windows are accumulated, the integrated average spectrum is displayed after each accumulation of 25 spectra. Since we have not, at this point, introduced error statistics in the computation of the individual ME spectra, the accumulated average spectrum is used as a more accurate guide to the stable resonance peaks. We retain a spectral resolution of $0.1 \mathrm{~Hz}$ for decade 2 and $1.0 \mathrm{~Hz}$ for decade 1 , throughout. The aim of the method is to simultaneously display time local and time averaged determinations of the resonance frequencies determined by the technique. Figure 6 shows this form of display for decade 1data for six successive accumulations of 25 data windows. For each set of 25 data windows (Fig. 6a-f), the 25 individual spectra are overlaid on the left and the accumulated average spectrum is 
shown on the right. Thus the accumulated average spectrum of Fig. $6 \mathrm{f}$ has been obtained using 150 successive data windows. Each of the six sets of 25 data windows shown in Fig. 6 correspond to a real time interval of $94 \mathrm{~s}$. A filter length $M=25$ was used throughout. The spectra shown in Fig. 6 define the $n=2-6$ order Schumann resonance peaks. The first order peak below $10 \mathrm{~Hz}$, although present, is not quantitatively resolved due to the high pass filter stage applied to data from this decade. Stable peaks are resolved at 14.0, 21.0, 27.0, 33.5 and $39.5 \mathrm{~Hz}$. The peak frequency and normalized power levels for the six accumulated average spectra are given in Table 2. The $\mathrm{n}=2-5$ stable peak frequencies compare well with other typical frequencies, such as 14.1, 20.3, 26.3 and $32.5 \mathrm{~Hz}$ (MADDEN and THOMSON, 1965).

Table 2. Resonance peak frequencies $[f(\mathrm{~Hz})]$ and the relative power levels $(P)$ of the $n=2-6$ Schumann resonance modes displayed in Fig. 6a-f

\begin{tabular}{ccccccccccc}
\hline & $f_{2}$ & $P_{2}$ & $f_{3}$ & $P_{3}$ & $f_{4}$ & $P_{4}$ & $f_{5}$ & $P_{5}$ & $f_{6}$ & $P_{6}$ \\
\hline (a) & 14.0 & 1.00 & 22.0 & 0.68 & 27.0 & 0.58 & 33.5 & 0.38 & 40.0 & 0.21 \\
(b) & 14.0 & 1.00 & 21.0 & 0.66 & 27.0 & 0.49 & 33.0 & 0.31 & 39.5 & 0.15 \\
(c) & 14.0 & 1.00 & 21.0 & 0.68 & 27.0 & 0.53 & 33.5 & 0.33 & 39.0 & 0.20 \\
(d) & 14.0 & 1.00 & 21.0 & 0.67 & 27.0 & 0.48 & 33.0 & 0.34 & 39.5 & 0.19 \\
(e) & 14.0 & 1.00 & 21.0 & 0.70 & 27.0 & 0.50 & 33.5 & 0.33 & 39.0 & 0.20 \\
(f) & 14.0 & 1.00 & 21.0 & 0.58 & 27.0 & 0.44 & 33.0 & 0.29 & 39.0 & 0.17 \\
\hline
\end{tabular}

When considering spectral details from decade 1 data some care should be exercised at frequencies of $50 \mathrm{~Hz}$ and above. Electromagnetic noise at such frequencies arises from the power distribution grid. The power-line voltage waveform is usually stable at $50 \mathrm{~Hz}$, however, the current waveform is often complicated and highly variable due to variable loads. Generally, power-line noise consists of steady spectral lines at the mains frequency and its odd harmonics, together with weaker spectral lines at sub-harmonic frequencies (e.g. $100 \mathrm{~Hz}$ ). In addition, the switching of current loads can produce broad-band transients and further high frequency harmonics. The $50 \mathrm{~Hz}$ notch filter mentioned previously has a centre frequency of $47.5 \mathrm{~Hz}$ and the attenuation achieved about this frequency is reflected in the spectra displayed in Fig. 6. A high frequency peak appears intermittently at around $97 \mathrm{~Hz}$ and is believed to be a power-line subharmonic. Other isolated large amplitude spectral components are occasionally detected at frequencies above $50 \mathrm{~Hz}$, as shown in Figs. $6 \mathrm{e}$ and $6 \mathrm{f}$. These are believed to be due to power-line transients.

There is an indication in the averaged spectral accumulations on the right of Fig. 6 that three higher order Schumann resonance modes $(n=9,10$ and 11$)$ are detected at frequencies around 53, 62 and $69 \mathrm{~Hz}$. The latter two modes were in fact the original experimental signatures of the Schumann resonances detected by BALSER and 
WAGNER (1960) and which were considered 'not statistically significant' at the time. The time local properties of the resonance frequencies are displayed as the overlaid plots on the left of Fig. 6 . These plots provide some indication of the spectral variability encountered when individual waveforms are considered. The detailed nature of these spectra is considered later, following a discussion of the sources of cavity excitation.

A similar exercise was conducted for decade 2 data in order to establish the characteristics of the first order Schumann resonance peak. Spectral plots, equivalent to those of Fig. 6 , are shown in Fig. 7 for a lower order $(M=12)$ filter length. This relatively low order of filter length was chosen to provide resolution of a single first order resonance peak for comparison with previous experimental determinations. Each of the six sets of 25 data windows corresponds to a real time interval of $262.5 \mathrm{~s}$. Despite the variability displayed in the over-laid plots of individual spectra, a stable resonance peak is obtained from the average spectra that vary in location between 7.50 and $7.65 \mathrm{~Hz}$, over the six accumulated sets of data windows. These figures for the first order mode appear slightly lower than typical published values of about $7.8 \mathrm{~Hz}$ (MADDEN and THOMSON, 1965; RYCROFT, 1965). However, as we have already noted in Fig. 4a, the single peak resolved for $M=12$ actually consists of two stable resonance peaks which can be distinguished by using higher order filter lengths. An equivalent plot for the first three sets of 25 data windows, obtained using $M=18$, is shown in Fig. 8. The two peaks are established at frequencies of (a) 7.3 and 8.0, (b) 7.25 and 8.1 ,(c) 7.25 and $8.1 \mathrm{~Hz}$. However, as can be seen in the overlaid data window plots, on the left of Fig. 8, individual spectra contain examples of both single and doublet Schumann resonance peaks. Such individual behaviour produces the varying contributions to the two resonance peaks defined in the average spectra of Figs. 8a, b and c. Such dynamic properties are better displayed using other methods of presentation, as discussed below.

The above results confirm that for the data set considered, the ME technique provides reliable time averaged estimates of the low order $(n=1-6)$ Schumann resonance modes. There is also an indication in Fig. 6 that the higher order Schumann resonance modes are detectable at this moderate latitude (i.e. $40^{\circ} \mathrm{N}$ ). In our opinion, however, the main advantage of the technique lies in the spectral resolution of individual waveforms which, according to the results presented, display complex frequency characteristics as a function of time. Many of these frequency characteristics are predicted by theory, as discussed below.

\section{DISCUSSION}


The digital AMT data considered in this study provide a bandwidth appropriate to studies of the Schumann resonance phenomena. The individual time windows are of the same order as the duration of many ELF events (e.g. Fig. 2).The study has demonstrated the possibilities that exist in the application of ME spectral techniques to such data. Using such techniques it appears that the spectral characteristics of individual Schumann resonance waveforms can be adequately resolved.

In the case of steady-state descriptions of the Earth-ionosphere waveguide properties, time-averaged characteristics may be required. The average spectra accumulated over time intervals of $94 \mathrm{~s}$ (decade 1 ) and $262.5 \mathrm{~s}$ (decade 2 ) shown in this study are examples of such properties. Although it is not our purpose here to investigate ionospheric parameters, the time averaged spectra of Figs. 6-8 would provide a basis for the evaluation of conductivity profiles according to the theory of TRAN and POLK (1979b). Within such accumulations time local variability is apparent, particularly in the case of the first order $(n=1)$ Schumann resonance mode. It has been demonstrated that the data for this mode contain time local frequency splitting. The waveguide appears to support the doublet frequencies of 7.3 and $8.1 \mathrm{~Hz}$. A sonogram displaying the largest resonance peaks for the first mode across approximately 6 min is shown in Fig. 9a. Because of the highly resolved nature of the spectra, a non-linear contour interval is required. The resolution inherent in Fig. 9a reveals the dynamic nature of the first order Schumann resonance mode. The largest resonance peak detected is indeed a doublet, however, well defined singlet peaks occur (e.g. RUN 42) and a possible triplet resonance is observed around RUN 29. The dynamic nature of the characteristics observed in Fig. 9a indicates that the time averaged properties of this mode must be treated with caution.

An equivalent sonogram displaying the largest resonance peaks for the $n=2,3,4$ and 5 and 6 Schumann resonance modes is shown in Fig. $9 \mathrm{~b}$. The time interval is restricted to approximately $3 \mathrm{~min}$ and again a non-linear contour interval has been used. The largest resonance peak occurs for the $\mathrm{n}=2$ mode and significant deviations from the average frequency of $14.0 \mathrm{~Hz}$ are observed. The same behaviour can be observed in the higher $(n=3,6)$ modes as well. There is also an indication (e.g. RUN 27) that the $n=3$ mode may comprise a doublet.

In order to understand the results presented, it is first necessary to consider the nature of the waveforms observed (e.g. Fig. 1), which are responsible for the spectra displayed in Fig. 9. From Fig. 1 we can distinguish two of the three possible signal components considered by BLIOKH et al. (1980, p. 121) to com- prise distinct sources of cavity 
excitation. The first component is the Schumann background, which is always present and which is the response of the cavity to continuous global lightning activity. The second category of signal component is referred to as an ELF transient event and typically exceeds the background by a factor of 2-10. According to JONES and KEMP (1971), the forms of such transient events may vary between a prolonged burst of complex field variations (e.g. Fig. 1, RUN 4) to a simple damped quasi- sinusoidal oscillation (e.g. Fig. 2). The two consecutive waveforms displayed in Fig. 2 possess the characteristic commencement of typical transient events noted by JONES and KEMP (1971, fig. 5). JONES and KEMP $(1970,1971)$ undertook both theoretical and experimental studies on such ELF events. In conclusion the authors suggest that such events are due to the transient excitation of cavity resonances by unusually large lightning discharges, the majority of which are cloud discharges.

The sonograms of Fig. 9 display the spectra associated with individual data runs. Obviously the resonances possessing the largest power densities arise from data runs containing larger than average transient events superimposed on the background. The lowest power densities arise from the continuum of back-ground sources. The sonograms of Fig. 9 necessarily emphasise the large amplitude transient events. We first consider the resonance characteristics for the $n=2,3,4,5$ and 6 modes displayed in Fig. 9b. According to JONES and KEMP (1970), the most important characteristic of the Schumann resonance spectrum is that, for a given source and fixed ionosphere, the spectral structure is a unique function of propagation distance. The theoretical computations on spectral behaviour as a function of distance from the source (JONES and KEMP, 1970, 1971) indicate an overall spectral structure that is strongly dependent on distance. We attribute much of the variability in resonance behaviour displayed in Fig. $9 \mathrm{~b}$ to such an effect. The main reason for this conclusion stems from the variety of horizontal magnetic field polarisation vectors displayed by the transient events. It is hoped to report on this work at a later date.

Turning now to the fine structure displayed by the first order mode in Fig. 9a. It is apparent that over relatively short periods of time (i.e. tens of seconds), the first order resonance mode is characterised by the presence of singlet, doublet and possibly triplet resonance peaks. We suggest that, since such characteristics are a pervasive feature of our data, they constitute both the split and non-split first order resonance modes predicted by theory and discussed in the introduction. We therefore attribute the observed fine structure to properties inherent in the cavity system, rather than to source-dependent characteristics. Such a conclusion requires that the mechanisms 
responsible for degeneracy within the cavity are highly dynamic over time scales of the order of minutes.

This initial empirical study is not exhaustive with regard to the available data. A small portion of the data set has been used to illustrate the use of a data adaptive spectral technique on electromagnetic data obtained at audio frequencies. The spectra resolution afforded by the technique is revealing and further work on the spectral and polarization properties of the waveforms is in progress.

\section{Acknowledgements}

The authors wish to thank a referee for useful advice and constructive comments. This paper is published with the approval of the Director, British Geological Survey (NERC). 


\section{REFERENCES}

AKaike $\mathrm{H}$.

AKAIKE $\mathbf{H}$

AKAIKE $\mathbf{H}$.

AKAIKE $\mathrm{H}$.

BALSER M. and WAGNer C. A.

BERRYMAN J. G.

Bliokh H., Nikolaenko A. P. and Filippov YU. F.

BURG J. P.

CANNON P. S. and Rycroft M. J.

FISCHER G.

Fougere P. F.

Galejs J.

GALEJS J.

GaleJs J.

JAYNES E.

Jenkins G. M. and WATTS D. G.

JONES D. L. and KEMP D. T.

JONES D. L. and KEMP D. T.

KELLER G. V.

LEVINSON $\mathrm{H}$.

MADDEN T. and THOMSON W.

PARZEN E

POLK C.

RYCROFT M. J.

SCHUMANN W. O.

SENTMAN D. D.

Strangway D. W., Swift S. M. JR and Holmer R. C.

TRAN A. and POLK C.

Tran A. and PolK C

Tran A. and Polk C.

ULRYCH T. J. and BISHOP T. N.

Ul.RYCH T. J. and ClaYton R. W.
1969

1970

1974

1976

1960

1978

1980

1968

1982

1982

1977

1961

1964

1972

1982

1968

1970

1971

1971

1947

1965

1976

1969

1965

1952

1983

1973

1976

1979a

$1979 b$

1975

1976
Ann. Inst. statist. Math., Tokyo 21, 407.

Ann. Inst. statist. Math., Tokyo 22, 203.

IEEE Trans. autom. Control AC-19, 716.

System Identification-Advances and Case Studies (Mehra R. K. and Lainiotis D. G., Eds). Academic Press, New York.

J. Res. natn. Bur. Stand. 64D, 415.

Geophysics 43, 1984.

Schumann Resonances in the Earth-Ionosphere Cavity (LlanWYN-JoNES D., Ed.), IEE Electromagnetic Wave Series 9. P. Peregrinus Ltd, Stevenage.

Paper presented at Advanced Study Institute on Signal Processing, NATO, Enschede, Netherlands.

J. atmos. terr. Phys. 44, 201

Geophys. Surv. 4, 373.

J. geophys. Res. 82, 1051.

IRE Trans. Antennas Propagn AP-9, 554.

Natural Electromagnetic Phenomena Below $30 \mathrm{kc} / \mathrm{s}$, (Blell D. F., Ed.). Plenum Press, New York.

Terrestrial Propagation of Long Electromagnetic Waves. Pergamon Press, New York.

Proc. IEEE 70, 939.

Spectral Analysis. Holden-Day, San Franscisco.

J. atmos. terr. Phys. 32, 1095.

J. atmos. terr. Phys. 33, 557.

Geoexploration 9, 99.

J. Math. Phys. 25, 261.

Rev. Geophys. 3, 211.

Technical Report 37, Statistical Science Division, State University of New York in Buffalo.

Planetary Electrodynamics, Vol. 2 (CORONITI S. C. and Hughes J., Eds). Gordon and Breach, New York.

J. Res. natn. Bur. Stand. 69D, 1071

Z. Naturf. 7A, 149.

J. atmos. terr. Phys. 45, 55.

Geophysics 38, 1159.

Radio Sci. 11, 803.

J. atmos. terr. Physl 41, 1241.

J. atmos. terr. Phys. 41, 1249.

Rev. Geophys. Space Phys. 13, 183.

Phys. Earth Planet. Interiors 12, 188. 


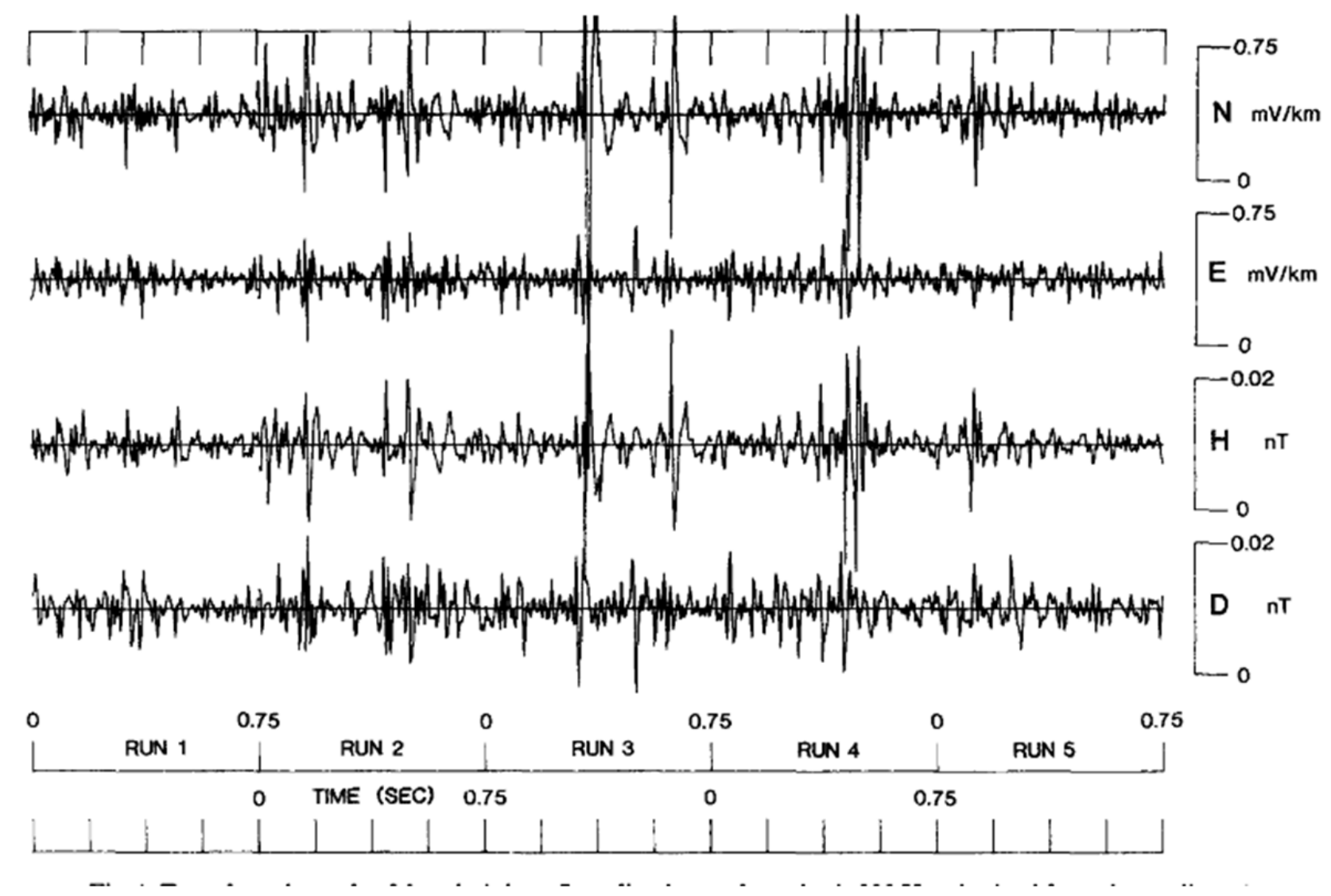

Figure 1. Four data channels of decade 1 data. Sampling interval on plot is $200 \mathrm{~Hz}$, obtained from data collected at 400 Hz. Five successive data windows of0.75 s length. $\mathrm{N}$ and $\mathrm{E}$ refer to 2 orthogonal components of the in- duced electric field. $H$ and $D$ are the corresponding magnetic $N-S$ and $E-W$ components. 

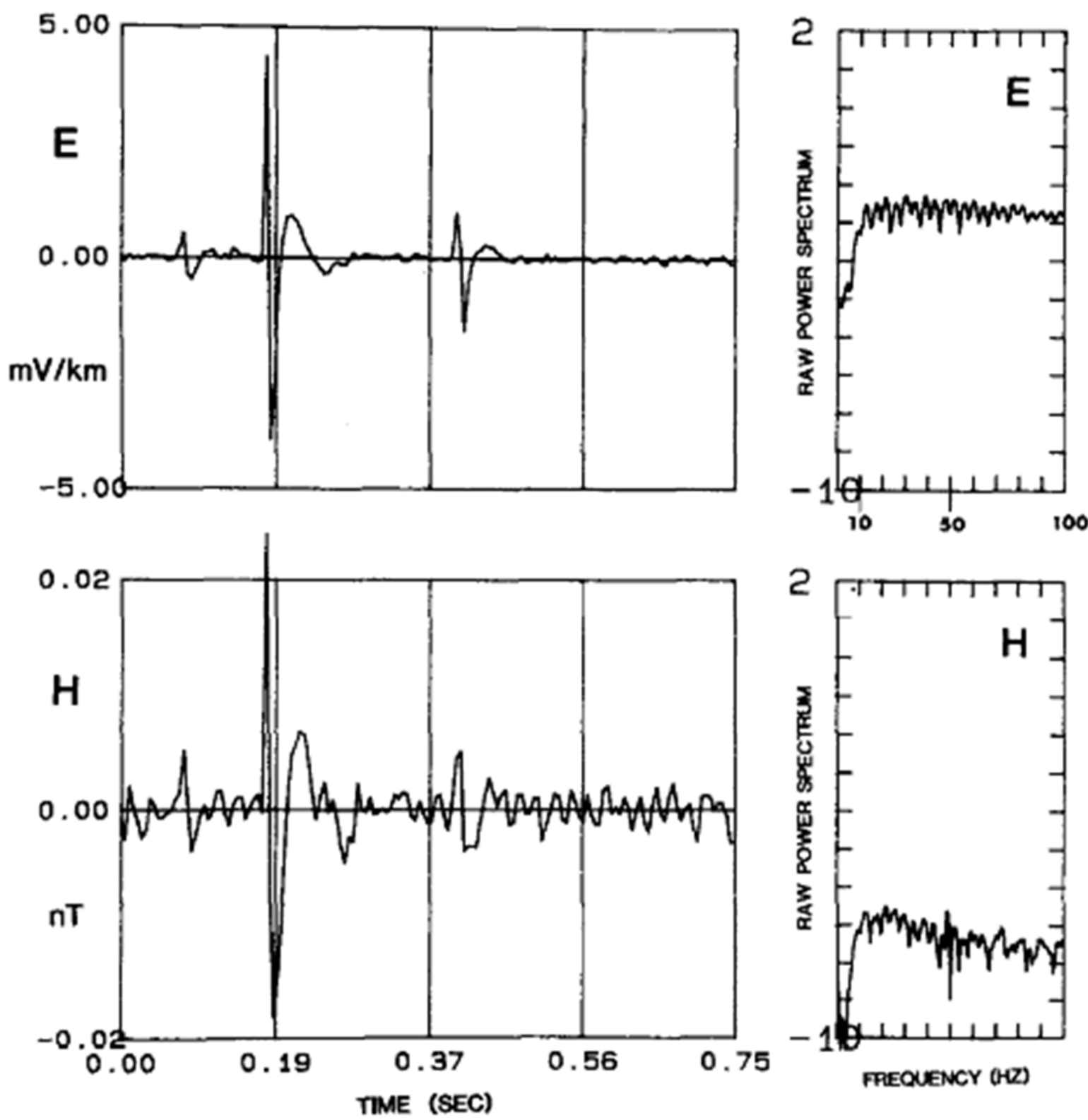

Figure 2. Data and raw power spectra from two channels of decade I data. $\mathrm{E}$ is the $\mathrm{E}-\mathrm{W}$ induced electric field, $\mathrm{H}$ is the magnetic N-S component. Raw spectra in units of $\left(m V . \mathrm{km}^{-1}\right)^{2} \mathrm{~Hz}^{-1}(\mathrm{E})$ and $(\mathrm{nT})^{2} \mathrm{~Hz}^{-1}(\mathrm{H})$. 

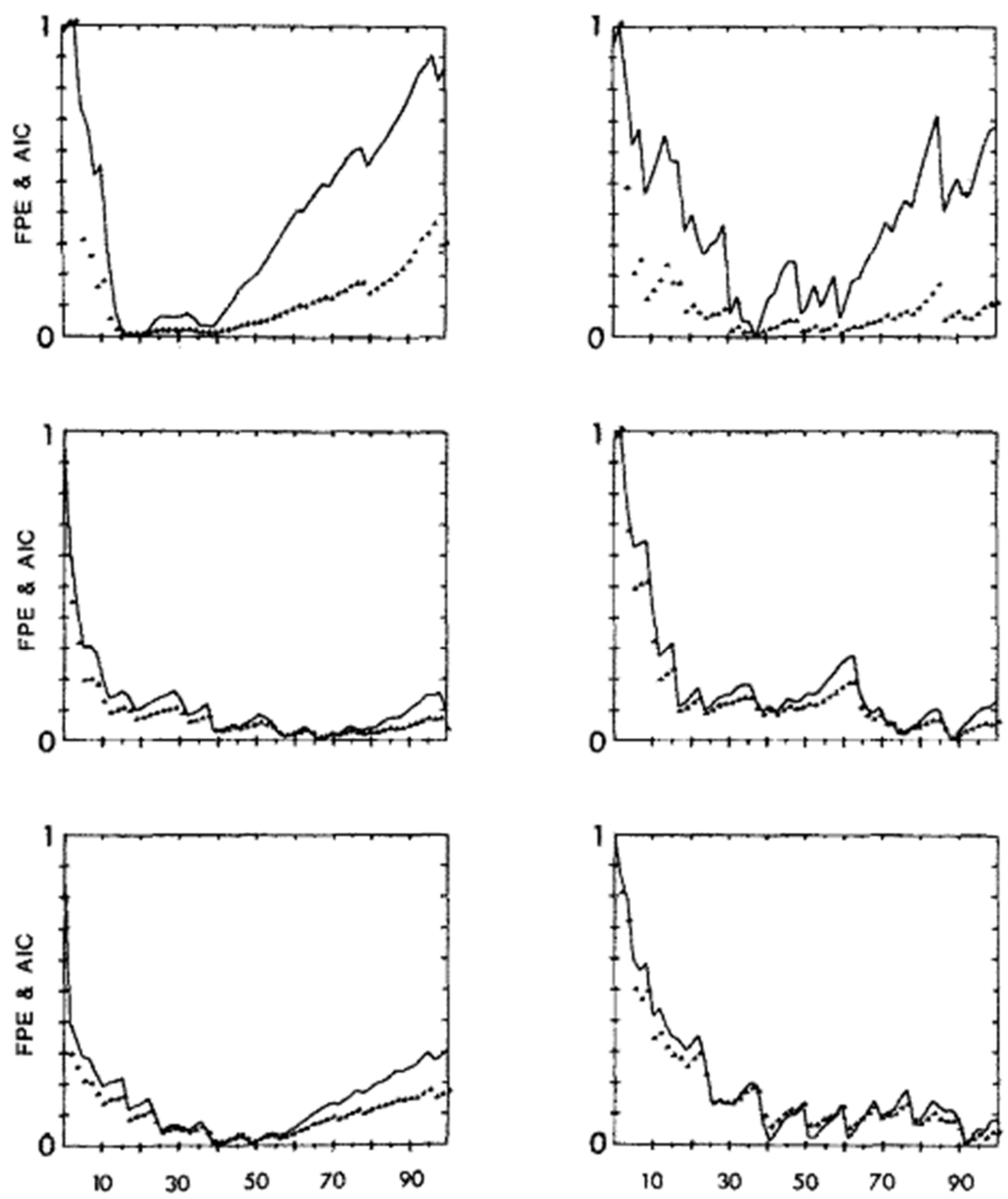

ORDER OF AR MODEL

ORDER OF AR MODEL

Figure 3a. Typical performance of two criteria for estimating filter length (order of AR model) on 6 windows of $H$ data. FPE, solid line, is Akaike's final prediction error. AIC, dotted line, is Akaike's theoretical information criterion. Values are normalized to a maximum value of unity. 

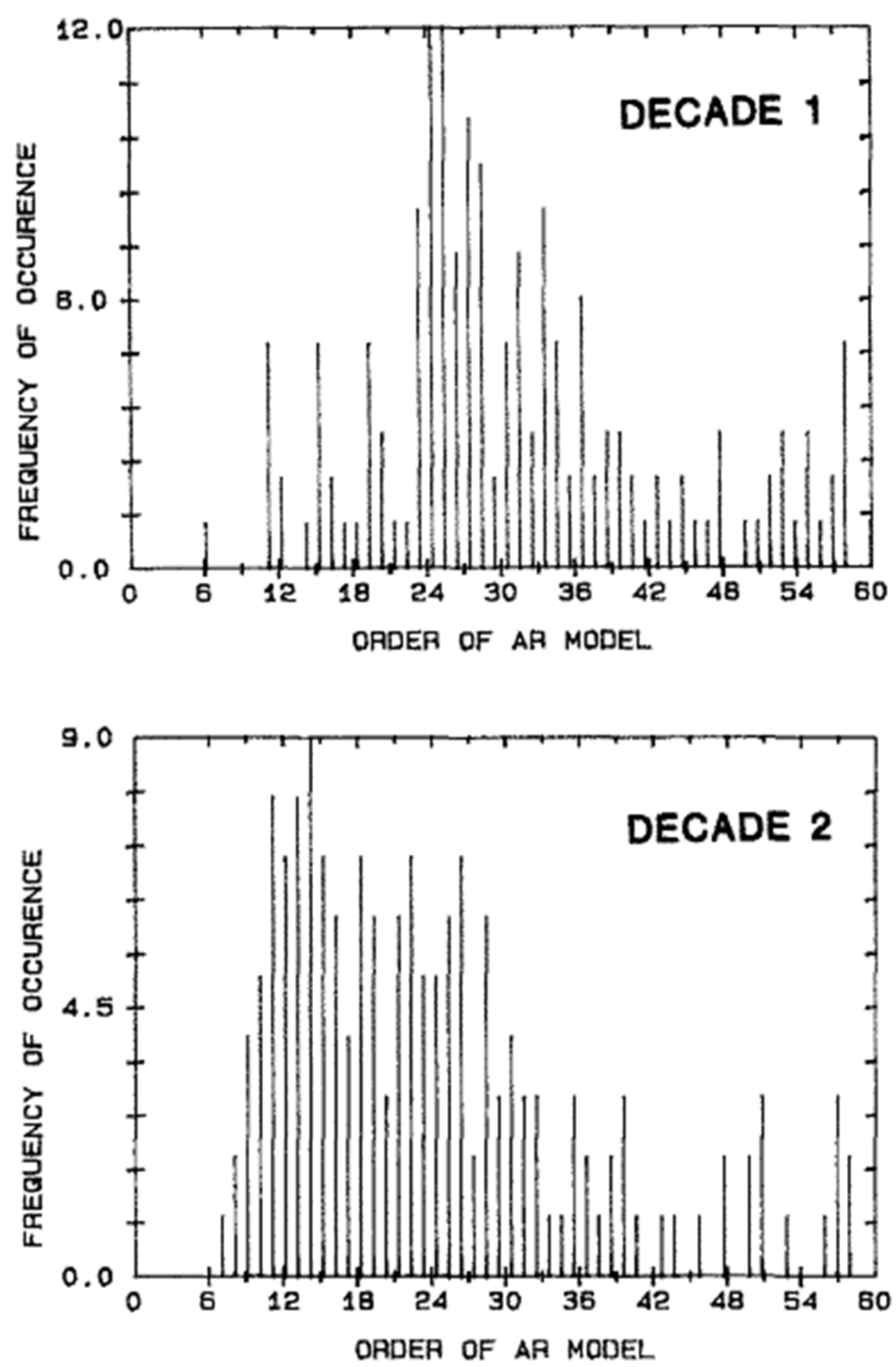

Figure 3b. Frequency of occurrence of the position of the absolute minimum of the FPE criterion asa function of the filter length (order of AR model) using I 50data runs of decade 1and decade 2 data. Filter lengths from 1 to 60 have been used. 
(a)
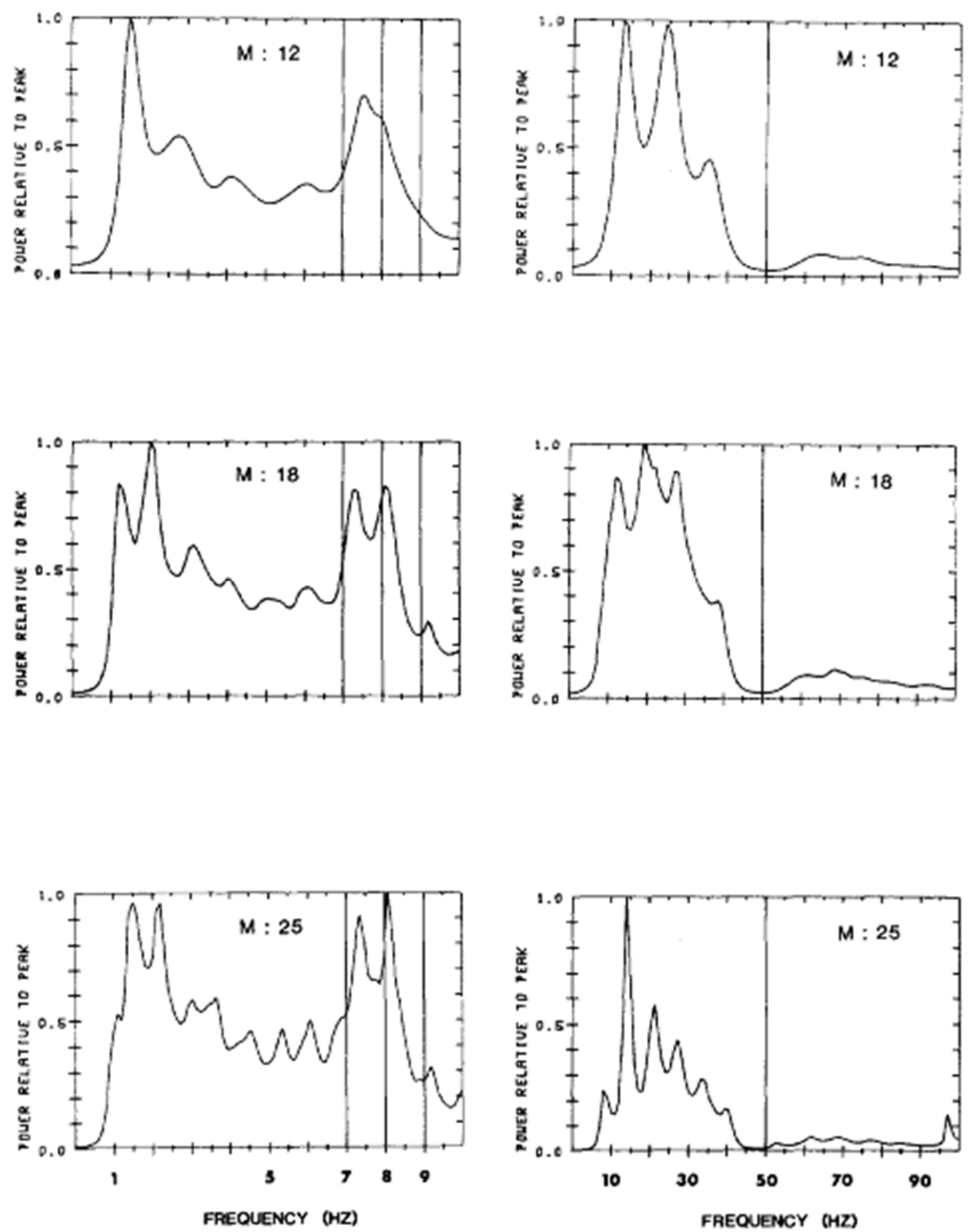

Figure 4. Effect of a change of filter length (M) on spectral resolution. Three cases are $M=12,18$ and 25. (a) Decade 2

data, 1-10 Hz; (b) decade 1 data, 10-100 Hz.
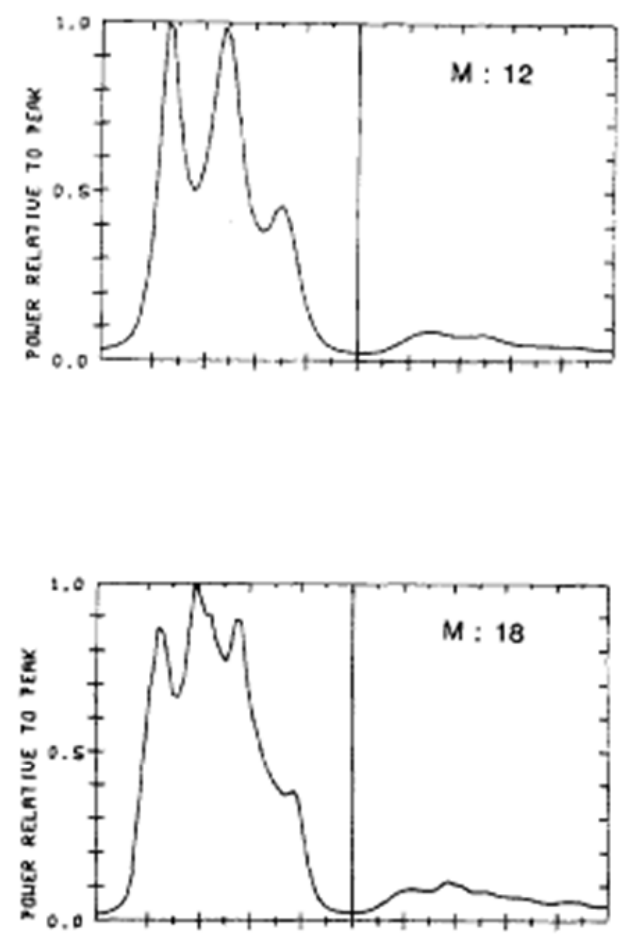

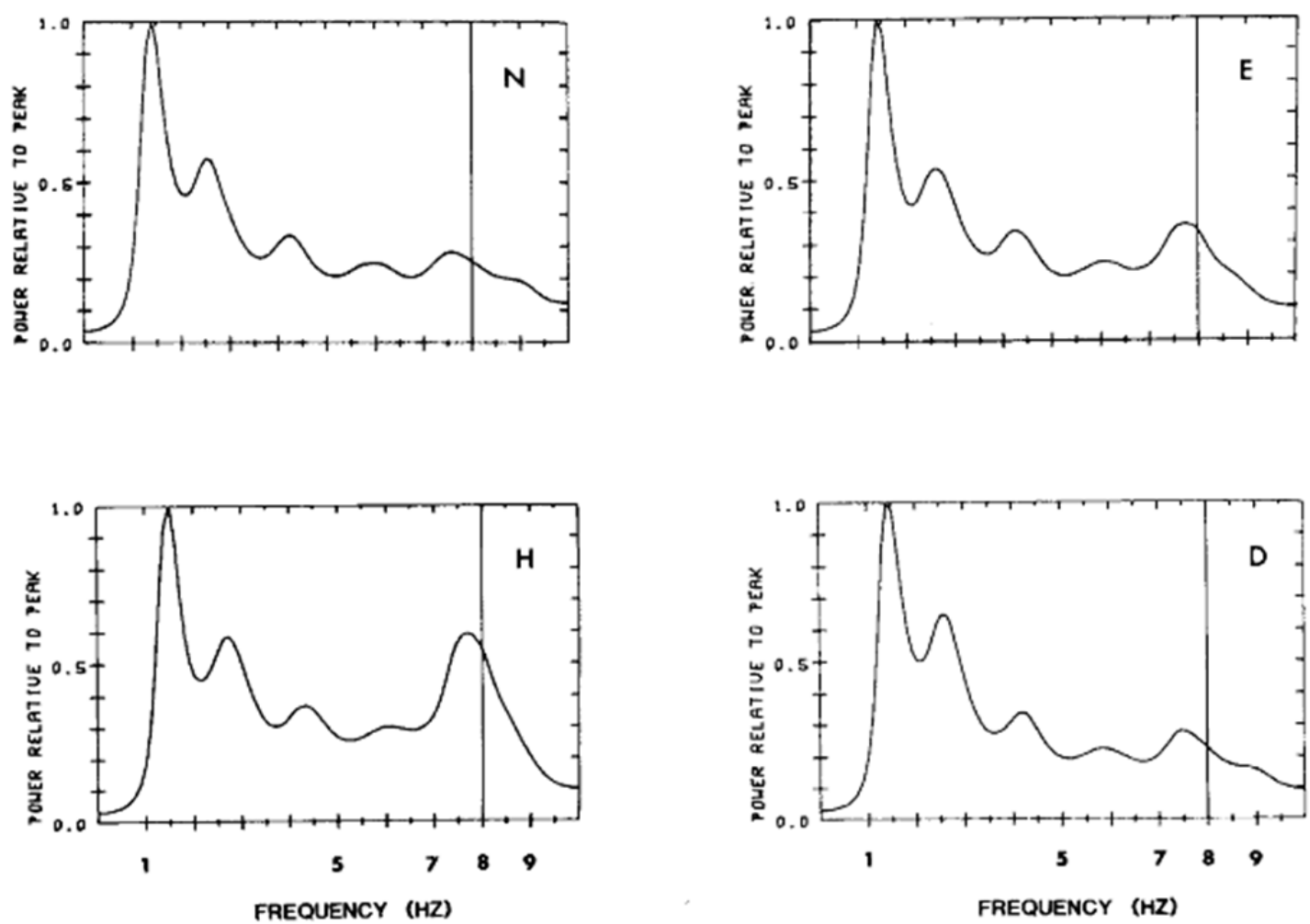

Figure 5. Equivalent spectral peaks in the 4 component data. Average spectra using a filter length of 12 of decade 2 data. $\mathrm{N}$ and $\mathrm{E}$ induced electric field. $\mathrm{H}$ and $\mathrm{D}$, magnetic field components. 


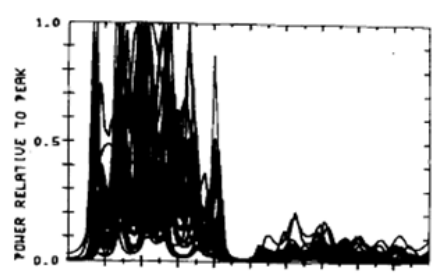

(a)
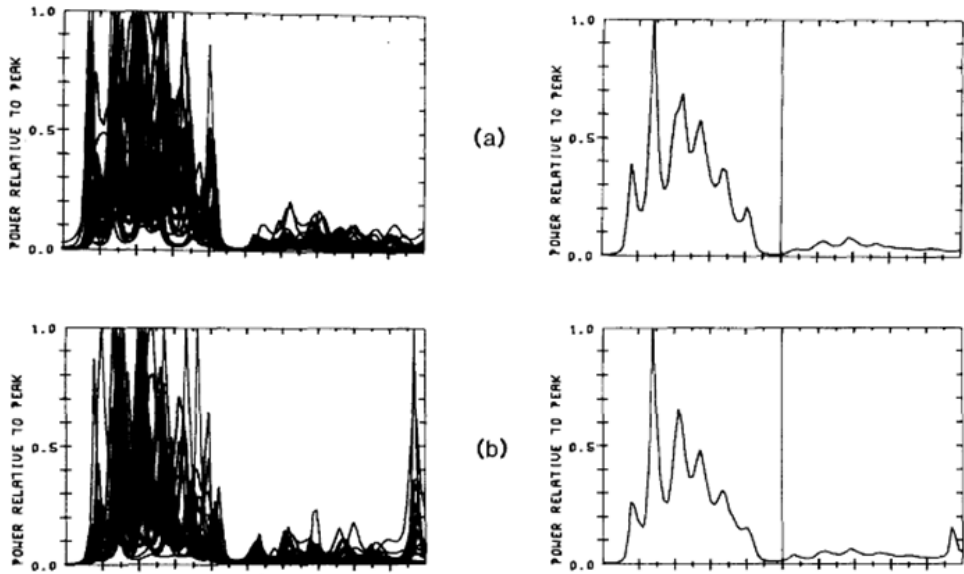

(b)
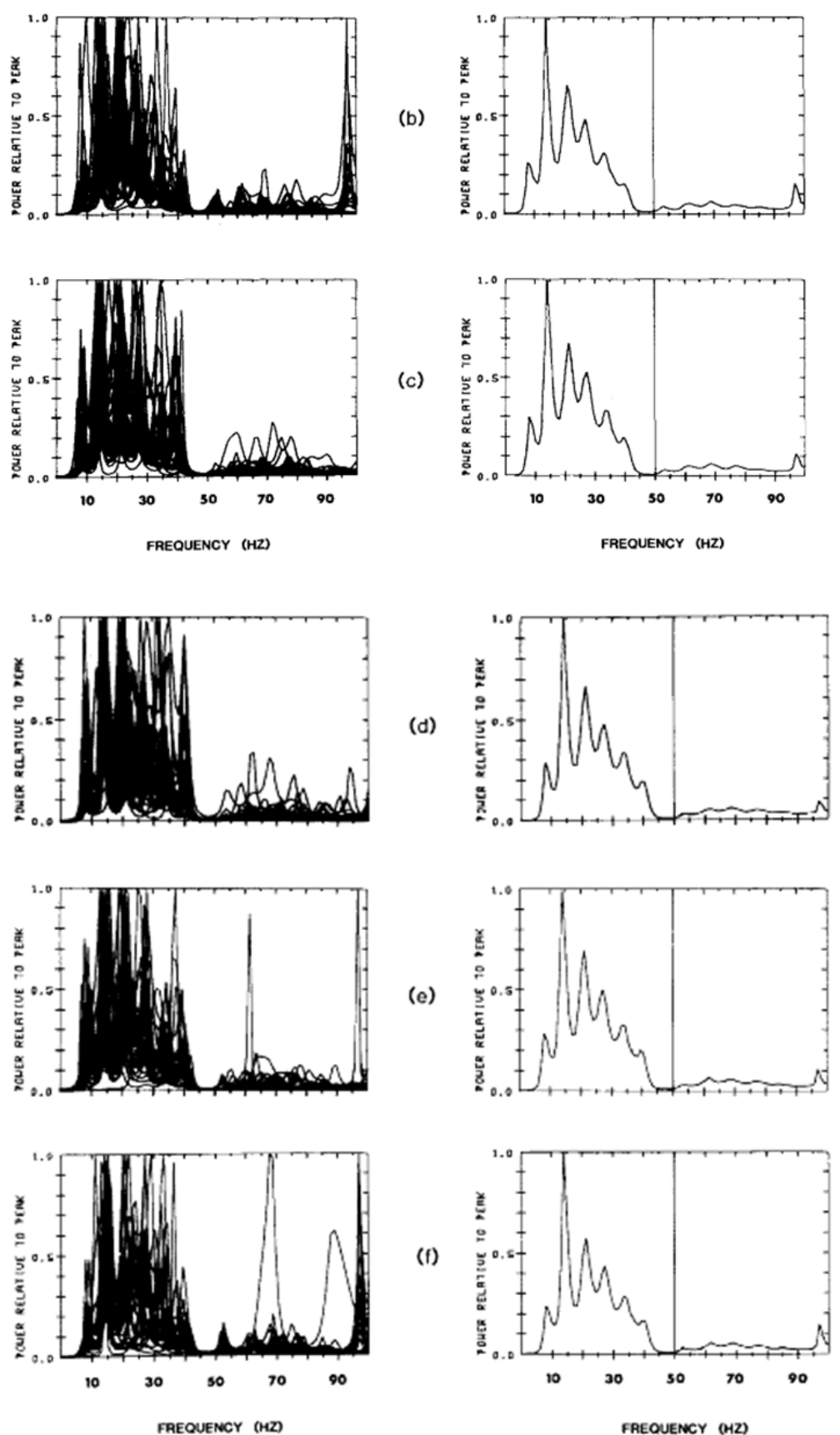

Figure 6. Individual (left) and average (right) spectra obtained from successive data accumulations of 25 data windows. Decade 1 data, $M=25$. Average spectra accumulate over all data windows. (a) Windows 1-25; (b) 26-50; (c) 51-75; (d) 76-100; (e) 101-125; (f) 126-150. 

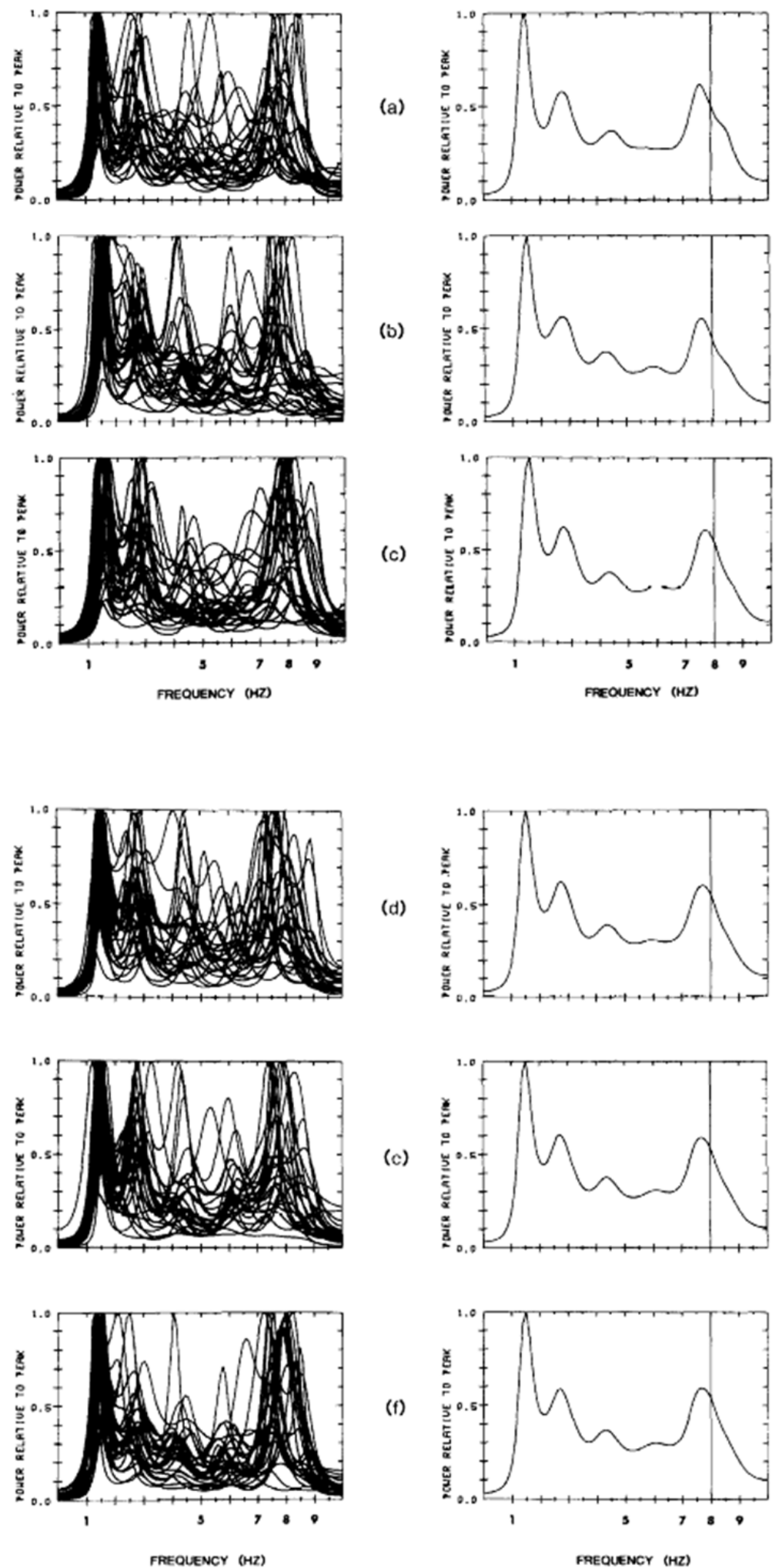

Figure 7. As per Fig. 6, but decade 2 data and $M=12$. 

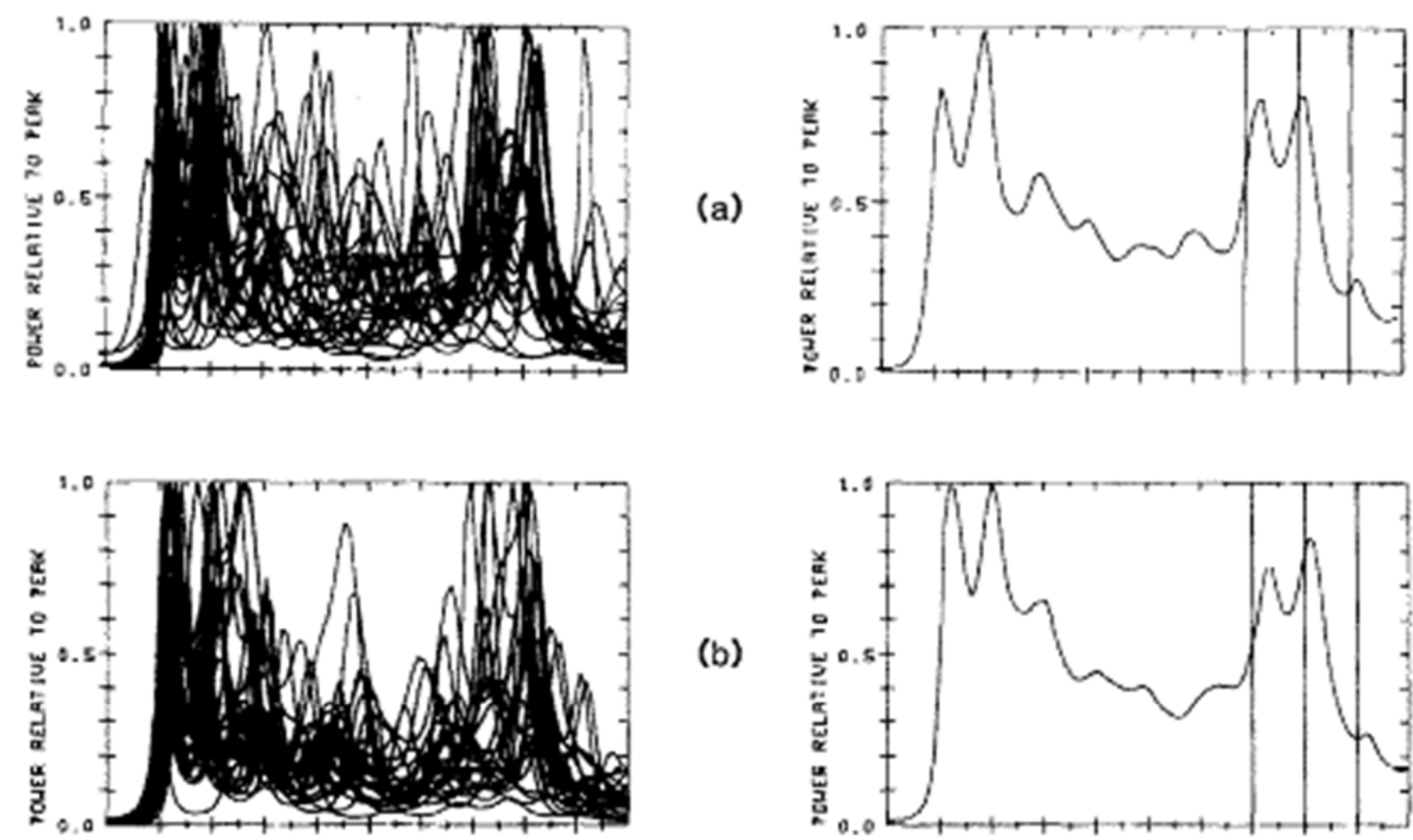

(b)
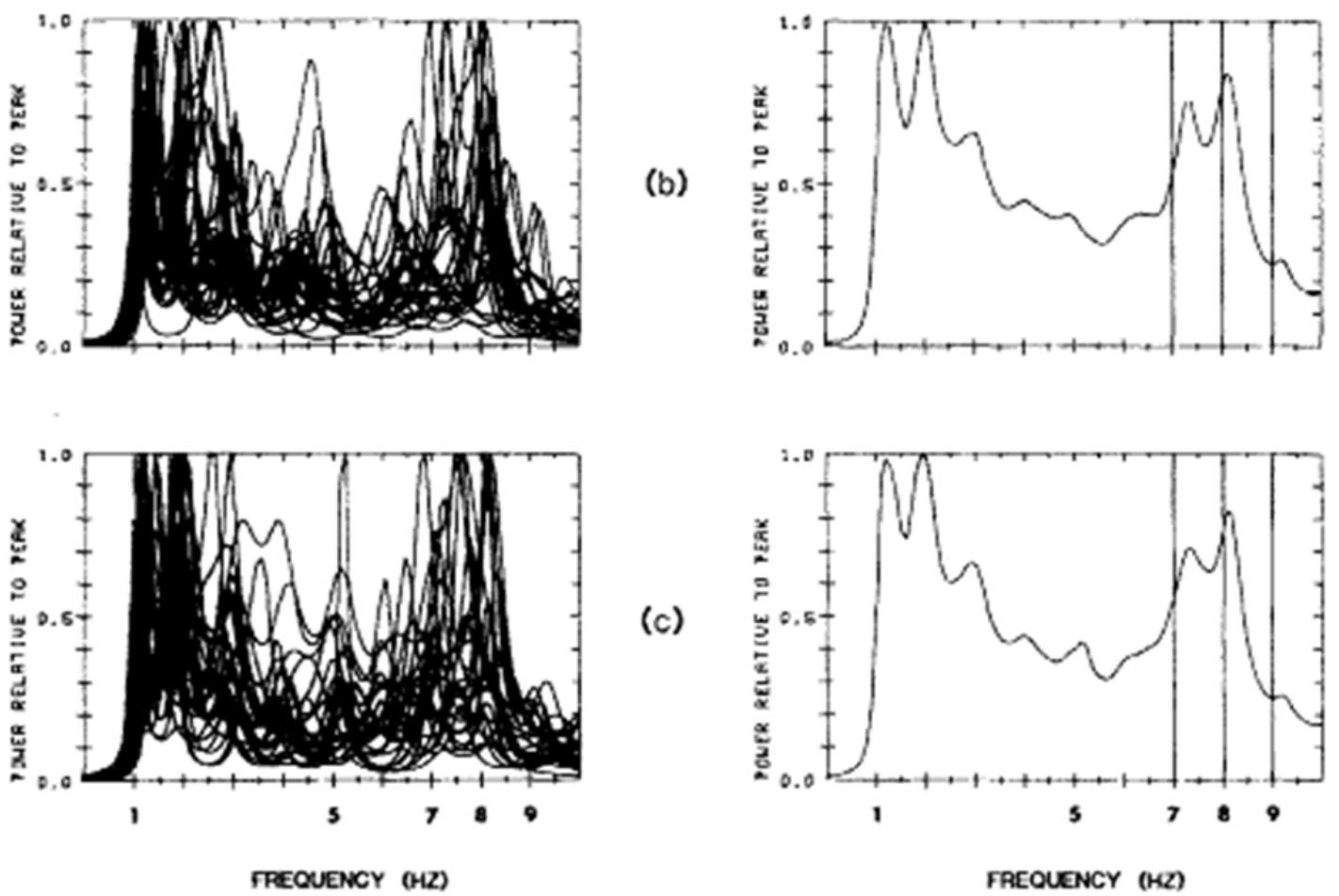

Figure 8. As per Fig. 6, but decade 2 data and $M=18$. (a) Windows 1-25; (b) 26-50; (c) 51-75 

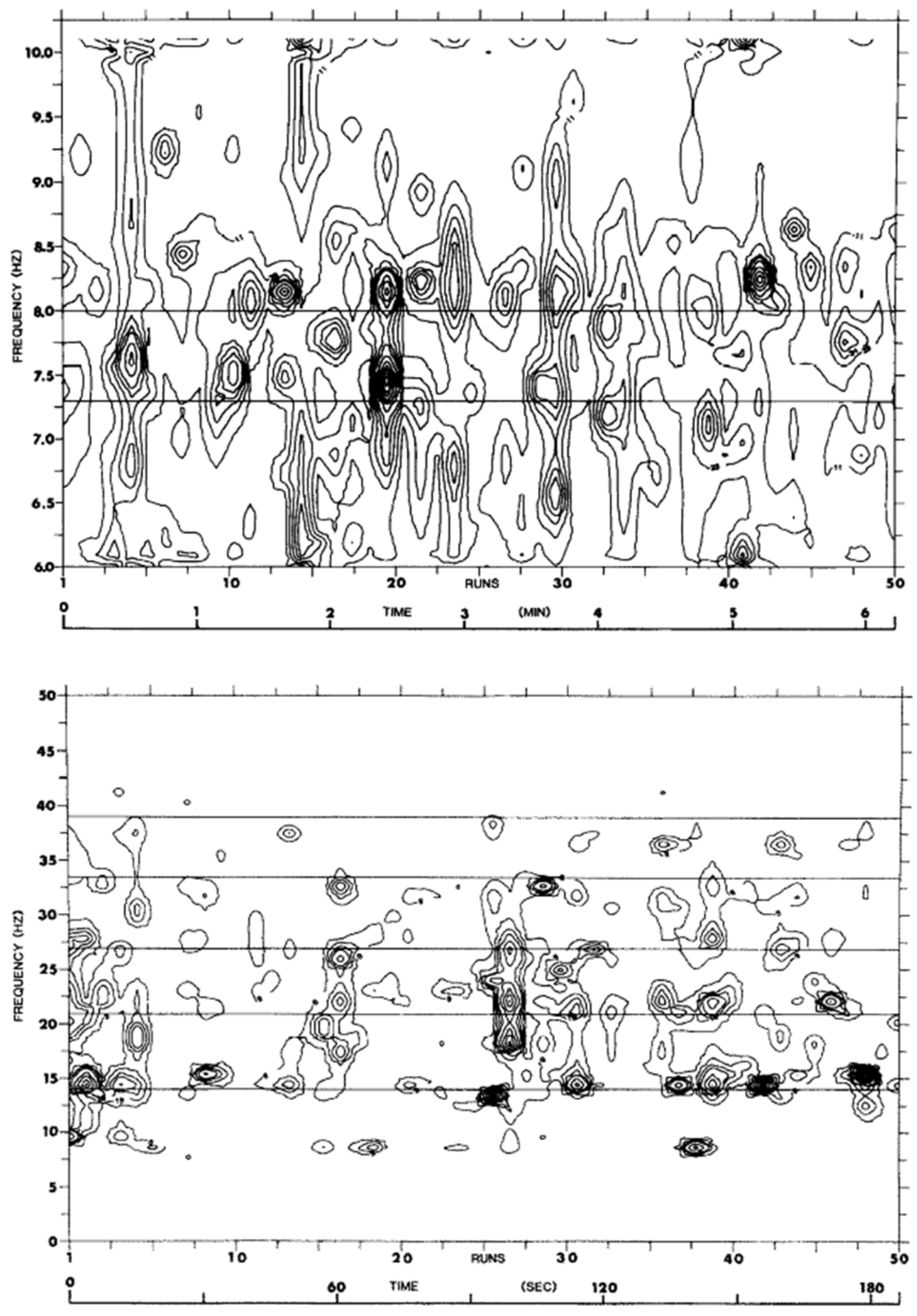

Figure 9. High resolution sonograms of the Schumann resonance modes. Power density values in $(\mathrm{nT})^{2} \mathrm{~Hz}^{-1} \times 10^{-8}$.Nonlinear contour interval with minimum value of $0.1 \times 10^{-10}$ (a) $n=1$ mode; decade 2 data; maximum value $0.23 \times 10^{-5}$; filter length $M=25$. (b) $n=2,3,4,5$ and 6 modes; decade 1 data; maximum value $0.15 \times 10^{-5}$; filter length $M=35$. 